\title{
Edge and fog services
}

\author{
Kuo-Ming Chao ${ }^{1} \cdot$ Jen-Yao Chung ${ }^{2}$
}

Received: 12 February 2019 / Accepted: 16 February 2019 / Published online: 2 March 2019

๑) Springer-Verlag London Ltd., part of Springer Nature 2019

Edge and fog computing is a new distributed computing paradigm [1] to meet increasing popularity of Internet of Things and distributed applications which can involve the generation of large volume and complex data sets, leading to complicated coordination among distributed processing and storage, etc. However, it could bring many benefits to the existing centralized systems by extending the scope of cloud computing, for example, to an end point of a network, allowing them to be more agile and dynamic, bringing resources close to end users or the places that happen to respond quickly and securely. The environment in which the edge or fog computing operates often includes heterogeneous computational resources or components that consist of diverse hardware and software systems (e.g., processing powers, storages, interfaces, operating systems, and programming languages) [2]. This hinders integration and interoperability of these resources. 5G and $\mathrm{AI}$ are two most prevailing trends in industry. Due to limited capacity of edges, embracing these two trendy technologies and exploiting them may present a challenge.

The reliability of resources in fog computing can be in question, due to the nature of its design and application. A network of edges or fog, for examples, could rely on wired or wireless network and be powered either by battery with various durations and limits, or by mains power. They may also be in an environment where they could be subject to interfere with communication. These issues are compounded by the varied sizes of resources that can be scattered and not even distributed. To efficiently utilize these relative dynamic and diverse resources require further study. Different communication protocols may be adopted in Internet of Things

\footnotetext{
Kuo-Ming Chao

k.chao@ coventry.ac.uk

Jen-Yao Chung

jy.chung@qct.io

1 Coventry University, Coventry, UK

2 QCT, San Jose, USA
}

(IoTs) and fog which could incur overheads and deter them in real-time applications.

A service model for fog computing could potentially address the above issues by introducing a new service-oriented architecture, demonstrating the advantage of cloud and distributing computing [3]. If edge and fog computing prevail, the reliability will need to be taken into accounts in the design of an innovative service model to improve its robustness. To design an enabling platform that migrates resources across networks seamlessly, optimize them, and maximize their utilization is an important research task to avoid resource underuse or overload. Introducing new highly efficient and reliable communication protocols to improve the performance and to meet the new requirements for edge and fog services is an urgent research topic to overcome the existing less competent ones. Security and privacy will be essential elements in new service models to gain the users' trusts.

SOA can advance and evolve edge and fog computing to meet the new demands, but it also brings a lot of challenges to the research communities. Here we only touch upon a few research issues and interests to draw community's attentions, so this is by no means an exhaustive list. We would like to invite researchers and practitioners from academic and industry to participate and contribute to this new emerging area by submitting their works on the following research topics, but not limited to.

- Resources optimization to alleviate bottlenecks

- QoS of fog computing

- Gateway/middleware for fog and cloud integration

- High-performance fog services

- Network topology for fog services

- Containers and virtualization of fog services

- Service middleware for edge and fog services

- New business model for edge and fog services

- Data and application migration in fog

- Service platform for edge and fog

- $5 \mathrm{G}$ for edge and fog services

- AI for edge and fog services

- Applications of edge and fog services 
In closing, the $5 \mathrm{G}$ and $\mathrm{AI}$ are two emerging areas of focus in industry and academic research communities. More research and innovation will contribute to the edge and fog services as the $5 \mathrm{G}$ and $\mathrm{AI}$ enable more of them to be moved to intelligent services with high-speed wireless communication that can change the perceptions and applications of edge and fog services. We perceive this as a rising opportunity, so we strongly encourage researchers to publish their works in the areas through SOCA.

\section{References}

1. Buyya R, Srirama S (2019) Fog and edge computing: principles and paradigms. Wiley Press, Hoboken
2. Verba N, Chao KM, James A, Goldsmith D, Fei X, Stan SD (2017) Platform as a service gateway for the fog of things. Adv Eng Inform 33:243-257

3. Sánchez M, Aguilar J, Exposito E (2018) Fog computing for the integration of agents and web services in an autonomic reflexive middleware. SOCA 12:333. https://doi.org/10.1007/s1176 1-018-0238-0

Publisher's Note Springer Nature remains neutral with regard to jurisdictional claims in published maps and institutional affiliations. 